\title{
Reversible left ventricular dysfunction associated with raised troponin I after subarachnoid haemorrhage does not preclude successful heart transplantation
}

\author{
E Deibert, V Aiyagari, M N Diringer
}

\begin{abstract}
Transient abnormalities in ECGs, echocardiograms, and cardiac enzymes have been described in the acute setting of subarachnoid haemorrhage. In addition, left ventricular dysfunction has been reported at the time of brain death. A patient with an acute subarachnoid haemorrhage who presented with raised troponin I (TnI) concentrations and diffuse left ventricular dysfunction is described. After declaration of brain death 32 hours later, the heart was felt initially not suitable for transplantation. A normal cardiac catheterisation, however, lead to successful transplantation of the donor heart. Raised catecholamine concentrations and metabolic perturbations have been proposed as the mechanisms leading to the cardiac dysfunction seen with brain death. This may be a biphasic process, allowing time for myocardial recovery and reversal of the left ventricular dysfunction. Awareness of this phenomenon in the acutely ill neurologic population needs to be raised in order to prevent the unnecessary rejection of donor hearts.

(Heart 2000;84:205-207)
\end{abstract}

Keywords: heart transplantation; troponin I; left ventricular dysfunction

Abnormal ECGs, cardiac arrhythmias, and

raised cardiac enzymes are common in intra-
cerebral haemorrhage, acute ischaemic stroke,

Neurology/Neurosurgery Intensive Care Unit, Department of Neurology, Box 8111, Washington University School of Medicine, 660 South Euclid Avenue, St Louis, MO 63110, USA E Deibert

V Aiyagari

M N Diringer

Correspondence to: Dr Ellen Deibert email: deiberte@ neuro.wustl.edu

Accepted 23 March 2000 subarachnoid haemorrhage, and severe head trauma. More recently, evidence of left ventricular dysfunction has been shown by echocardiography. An important and often unrecognised characteristic of these abnormalities is that they are reversible in the setting of subarachnoid haemorrhage.

We report a case of a patient who suffered a subarachnoid haemorrhage and later progressed to brain death. Despite concerns over raised troponin I ( $\mathrm{TnI}$ ) concentrations and poor cardiac function before brain death, further cardiac evaluation found the heart to be functioning normally and suitable for transplant.

\section{Case report}

A 51 year old African American female transiently lost consciousness and on arrival to the emergency department, complained of severe occipital headache with blurred vision. Her Glasgow coma scale was 14 and she had mild right sided facial weakness. Past medical history included tobacco use (half pack per day for 30 years). There was no prior history of diabetes, hypertension, coronary artery disease, cardiac arrhythmias, or chest pain. Computed tomography of the head revealed diffuse subarachnoid haemorrhage with intraventricular extension and mild hydrocephalus. A large right vertebral artery aneurysm was discovered on cerebral angiography.

Moderate pulmonary oedema was present on chest radiogram and the ECG was abnormal, demonstrating sinus tachycardia (106 beat per minute), a PR interval of $152 \mathrm{~ms}$, a QRS duration of $92 \mathrm{~ms}$, a prolonged QTc interval of $489 \mathrm{~ms}$, and a QRS axis of $67^{\circ}$. Flattened and inverted $\mathrm{T}$ waves were seen in leads I and AVL respectively. Total creatine kinase (CK), CK-MB fraction, and TnI values are presented in table 1. Echocardiogram on admission revealed global hypokinesis with an estimated left ventricular ejection fraction of $40 \%$.

Approximately 24 hours after the initial subarachnoid haemorrhage, the patient became unresponsive and within a few hours lost all signs of brain function. She was pronounced dead based upon neurologic criteria, approximately 32 hours after her initial ictus. The patient's family requested organ donation.

Because of the results of the cardiac evaluation on admission, the heart was not considered suitable for transplantation. However, cardiac catheterisation performed 12 hours after time of death revealed normal left ventricular function with normal coronary arteries, and the decision was reversed.

Table 1 Total CK, CK-MB fraction, and troponin I concentrations of patient before organ donation

\begin{tabular}{llll}
\hline Laboratory test & $\begin{array}{l}0-12 \\
\text { hours }\end{array}$ & $\begin{array}{l}13-24 \\
\text { hours }\end{array}$ & $\begin{array}{l}25-36 \\
\text { hours }\end{array}$ \\
\hline $\mathrm{CK}$ total $\mathrm{u} / \mathrm{l} /(\mathrm{MB}$ fraction $\mathrm{ng} / \mathrm{ml})$ & $145 /(6)$ & $191 /(6)$ & $125 /(5)$ \\
Troponin I $\mathrm{ng} / \mathrm{ml}$ & 2.3 & 3.5 & 3.2 \\
\hline
\end{tabular}

$\mathrm{CK}$, creatine kinase; $\mathrm{CK}-\mathrm{MB}, \mathrm{MB}$ isoenzyme of creatine kinase. 


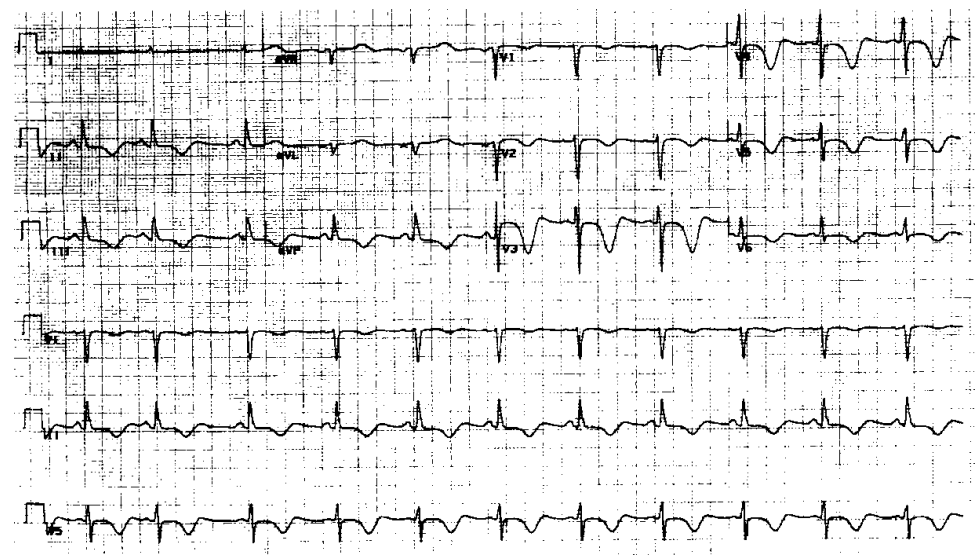

Figure 1 EGG recorded within 24 hours of onset of subarachnoid haemorrhage. Note the inverted $T$ waves in leads II, III, AVF, V2, V3, V4, V5, and V6. In addition, there is ST segment elevation in V3, V4, and V5. These findings are common ECG abnormalities following subarachnoid haemorrhage.

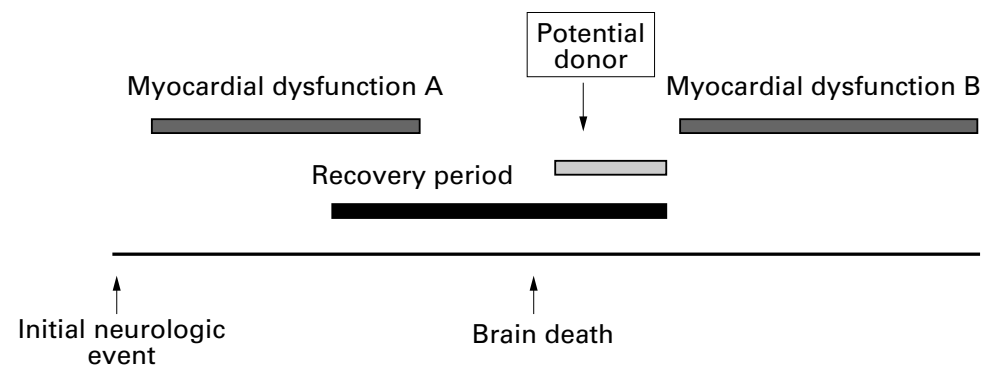

Figure 2 As proposed by Novitzky, myocardial dysfunction at the time of brain death may consist of two physiologic processes. ${ }^{9}$ An initial injury is caused by a high catecholamine state (myocardial dysfunction $A$ ), and a second injury is induced by metabolic perturbations (myocardial dysfunction B). We propose that these two processes do not necessarily occur contiguously, but instead occur as a biphasic phenomenon allowing for a period of myocardial recovery. If appropriate haemodynamic support is given, the second metabolic induced injury could be delayed, allowing a window of opportunity for successful organ procurement.

The heart was successfully transplanted to a 60 year old woman with severe idiopathic cardiomyopathy. The surgery was uneventful and the recipient made a complete recovery. An echocardiogram performed nine months after transplant revealed normal right ventricular and left ventricular function, normal left ventricular/right ventricular wall thickness, a mildly dilated left ventricle, biatrial enlargement, mild mitral regurgitation, moderate tricuspid regurgitation, mild pulmonary insufficiency, and a pulmonary artery pressure of $35 \mathrm{~mm} \mathrm{Hg}$. There was no pathological evidence for rejection on an endomyocardial biopsy performed at the same time.

\section{Discussion}

This case illustrates the reversible nature of left ventricular dysfunction following subarachnoid haemorrhage, despite progression to brain death. Cardiovascular abnormalities are well described following non-traumatic subarachnoid haemorrhage. ECG abnormalities, such as depressed or elevated ST segments, prominent $\mathrm{U}$ waves, QT prolongation, and peaked or inverted $\mathrm{T}$ waves, (see example in fig 1) are observed in $50-90 \%$ of cases. Most occur within the first 72 hours after subarachnoid haemorrhage. ${ }^{1}$ Only $4 \%$ of the patients have malignant arrhythmias such as ventricular tachycardia, torsades, and asystole. ${ }^{2}$ In addition, cardiac markers such as $\mathrm{CK}-\mathrm{MB}$ and $\mathrm{TnI}$ are raised in subarachnoid haemorrhage patients. ${ }^{3}$

More recently, reversible left ventricular dysfunction has been described after acute subarachnoid haemorrhage. For instance, 7/12 patients evaluated within 12 hours of subarachnoid haemorrhage had reduced wall motion of the left ventricular apex on echocardiogram, which improved on repeat study 2-3 weeks later. ${ }^{4}$ None had any significant angiographic coronary artery disease. Another study found that $8 \%(5 / 57)$ of subarachnoid haemorrhage patients had wall motion abnormalities on echocardiogram. ${ }^{5}$ Four of these patients had repeat echocardiograms in which function had returned to normal.

The cause of left ventricular dysfunction after subarachnoid haemorrhage is not known. The prevailing theory is that it is caused by increased sympathetic activity, either via the adrenal medullary axis, or increased activation of the sympathetic innervation of the heart. Subarachnoid hemorrhage patients with raised catecholamine concentrations on presentation are more likely to have ECG abnormalities. ${ }^{6}$ Necropsies show patchy areas with cardiac myofibrillar degeneration, a distinct pathological entity from coagulation necrosis seen with ischemic coronary artery disease. ${ }^{7}$ Although evidence suggests that the pathophysiology is not coronary artery disease, the exact mechanism and the reason for reversibility have yet to be elucidated.

On the other hand, left ventricular dysfunction seen at the time of brain death is well described and believed to be caused by a combination of two separate physiologic processes. ${ }^{89}$ First, there is a release of catecholamines at the time of the initial neurologic insult. The second event is related to several metabolic perturbations. Thyronine, thyroxine, cortisol, antidiruretic hormone, and insulin concentrations are decreased at the time of brain death. Eventually, aerobic metabolism is believed to cease, creating increased tissue acidosis and loss of myocardial energy stores. ${ }^{9}$ The administration of tri-iodothyronine has been shown to be useful in salvaging donor hearts with decreased left ventricular function and returning to aerobic metabolism. ${ }^{9}$

It seems plausible that the reversible left ventricular dysfunction seen in acute subarachnoid haemorrhage is similar to that seen at the time of brain death. What is not recognised is that the above processes do not need to occur contiguously, allowing for a period of recovery before the onset of the metabolic injury (fig 2). It is possible that our patient suffered catecholamine induced left ventricular dysfunction on the day of her subarachnoid haemorrhage and had enough time for myocardial recovery before her second haemorrhage that resulted in brain death. With appropriate haemodynamic support the heart remained suitable for transplantation. Thus, a window of opportunity may occur between the neural and humoral components of this apparent biphasic process, that allows for left ventricular recovery and the potential for a new donor heart.

In addition, current cardiac enzyme assays are both sensitive and specific for myocardial 
injury, but in this population it is not clear as to what degree the myocardium has been injured or if it is permanent. Until further work can be done to quantify better these assays in the neurologic population, we recommend a thorough cardiac re-evaluation at the time of brain death in all patients suffering from left ventricular dysfunction at the time of their acute subarachnoid haemorrhage. A cardiac catheterisation is essential to determine the true state of coronary arteries in this population, and to avoid unnecessary rejection of potential heart donors.

1 Brouwers PJAM, Wijdicks EFM, Hasan D, et al. Serial electrocardiographic recording in aneurysmal subarachnoid trocardiographic recording in aneurys

2 Di Pasquale G, Pinelli G, Andreoli A, et al. Holter detection of cardiac arrhythmias in intracranial subarachnoid hemorrhage. Am f Cardiol 1987;59:596-600. 3 Horowitz MB, Willet D, Keffer J. The use of cardiac
troponin I (cTnI) to determine the incidence of myocardial troponin I (cTnI) to determine the incidence of myocardial
ischemia and injury in patients with aneurysmal and ischemia and injury in patients with aneurysmal and presumed aneurysmal subar

4 Kono T, Morita H, Kuroiwa $\mathrm{T}$, et al. Left ventricular wall motion abnormalities in patients with subarachnoid hemorrhage: neurogenic stunned myocardium. $\mathcal{F} \mathrm{Am}$ Coll Cardiol 1994;24:636-40.

5 Mayer S, Limandri G, Sherman D, et al. Electrocardiographic markers of abnormal left ventricular wall motion in acute subarachnoid hemorrhage. f Neurosurg 1995;83:88996.

6 Cruickshank JM, Neil-Dwyer G, Stott AW. Possible role of catecholamines, corticosteroid, and potassium in production of electrocardiographic abnormalities associated with tion of electrocardiographic abnormalities associated with

subarachnoid hemorrhage. Br Heart f 1974;36:697-706. Neil-Dwyer G, Cruickshank JM, Doshi R. The stress response in subarachnoid hemorrhage

8 Seiler C, Laske A, Gallino A, et al. Echocardiographic evaluation of left ventricular wall motion before and after heart transplantation. F Heart Lung Transplant 1992;11:867-74.

9 Novitzky, D. Novel actions of thyroid hormone: the role of triiodothyronine in cardiac transplantation. Thyroid 1996; 6:531-6.

\section{IMAGES IN CARDIOLOGY}

\section{Thromboembolic leg ischaemia as first manifestation of an unrecognised myocardial infarction}

A 39 year old previously healthy man presented with a painful, cold, and pulseless right leg. His past medical history was uneventful. In particular, the patient had never experienced chest pain. Upon questioning, he remembered that three months earlier he had suffered intermittent right shoulder pain with irradiation to the right arm lasting a few days. Subsequently, he noticed a decreased exercise capacity. Routine ECG showed Q waves and ST segment elevation in the precordial leads suggestive of prior anterior myocardial infarction with left ventricular aneurysm. Echocardiography (left) confirmed the diagnosis, demonstrating an apical aneurysm and a large apical mural thrombus (arrow) (RA, right atrium; RV, right ventricle; LA, left atrium; LV, left ventricle).
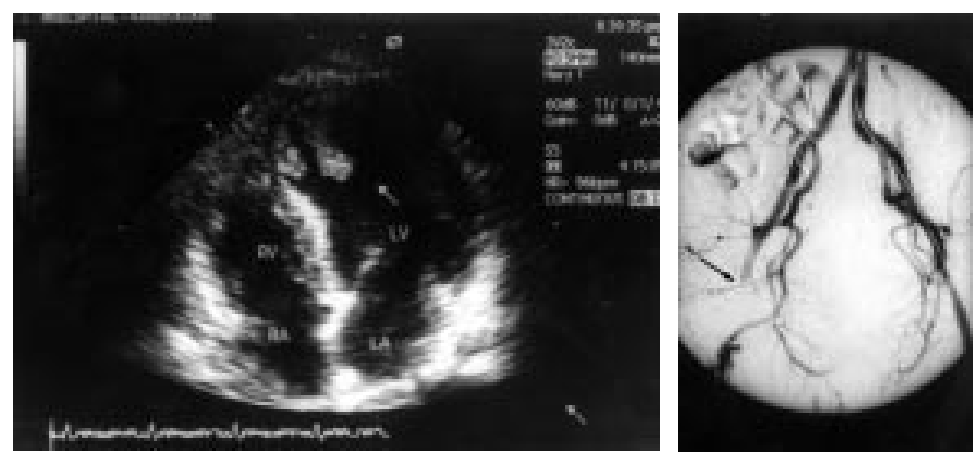

Lower extremity digital subtraction angiography (right) showed multiple thromboembolic occlusions of the right femoral bifurcation, the right superficial femoral artery, and the right popliteal artery with the classical "meniscus" sign (arrow). The absence of peripheral vascular disease, the involvement of the femoral bifurcation, the multiple localisation of the occlusions, and the clear cut-off of the contrast dye are typical angiographic signs of embolic occlusions.

Surgical embolectomy was performed. Elective coronary angiography showed a tight stenosis at the bifurcation of the left anterior descending coronary artery with the first diagonal branch. This lesion was successfully treated by percutaneous transluminal coronary angioplasty. Postprocedural treatment included aspirin and coumadin. At the three month follow up there was no evidence of further embolic events.

Our case illustrates that, even in young patients, owing to its atypical or silent presentation, a large myocardial infarction may go unrecognised and manifest itself only by the occurrence of an infarct related complication.

MARCO ROFFI

FELIX MAHLER FRANZ R EBERLI 